\title{
Dipolar anisotropy in quasi-2D honeycomb antiferromagnet $\mathrm{MnPS}_{3}$
}

\section{(The original publication is available at www.epj.org)}

\section{J. Goossens}

Research School of Chemistry, The Australian National University, Canberra 0200, Australia

Received: 08/09/10 / Revised version: 30/10/10

\begin{abstract}
The nature of the anisotropy in magnetic systems which show isotropic Heisenberg exchange is crucial in determining their magnetic properties. This is particularly true in low-dimensional systems in which the very existence of long-range order depends on the anisotropy. The honeycomb lattice $\mathrm{MnPS}_{3}$ system has been studied as an example of a magnetically quasi-two-dimensional system of unusual symmetry. In this paper the effect of the dipole-dipole interaction in $\mathrm{MnPS}_{3}$ on the magnetic ordering is explored through modelling. It is found that the dipolar anisotropy can explain the spin directions both in zero field and above the spin flop phase transition, but it is important that real rather than idealised atomic coordinates are used; this latter consideration is significant because in performing theoretical calculations, it may sometimes be assumed that small deviations away from the ideal can be ignored, but in truth they determine key aspects of the behaviour.
\end{abstract}

\section{Introduction}

Low-dimensional magnetism allows theoretically tractable models to be realised experimentally, for example the Onsager solution to the $2 \mathrm{D}$ (two-dimensional) rectangular Ising model [1]. Much of the study has centred on systems such as $X_{2} Y \mathrm{~F}_{4}(X=\mathrm{Rb}$ or $\mathrm{K}$ and $Y=\mathrm{Mn}$, Co or $\mathrm{Ni}$ for example $[2,3]$ and on the $\mathrm{Cu}-\mathrm{O}$ planes in high temperature superconductors and their parent compounds [4]. In these materials the $2 \mathrm{D}$ lattice is square and the $c$ axis is perpendicular to the $2 \mathrm{D}$ magnetic planes, unlike in $\mathrm{MnPS}_{3}$.

$\mathrm{MnPS}_{3}$ is a layered quasi-two-dimensional Heisenberg antiferromagnet on a honeycomb lattice. The structure is monoclinic and the in-plane coordination number is 3 , meaning that $\mathrm{MnPS}_{3}$ is a system whose symmetries are quite different from those of other $2 \mathrm{D}$ magnetic materials. It has been studied in the context of hydrogen sorption [5], fundamental magnetism [6], and a range of intercalation reactions, including the effect of intercalation on magnetism [7-9].

The unit cell parameters are $a=6.077 \AA, b=10.524$ $\AA, c=6.769 \AA$ and $\beta=107.35^{\circ}[10]$. The space group is $C 2 / m$. The $\mathrm{Mn}^{2+}$ ions lie at fractional coordinates $(0, y, 0)$, $\left(\frac{1}{2}, \frac{1}{2}-y, 0\right),(0,1-y, 0)$ and $\left(\frac{1}{2}, \frac{1}{2}+y, 0\right)$ with $y_{\mathrm{Mn}} \sim \frac{1}{3}$. The crystal structure is shown in figure 1 and shows the honeycomb arrangement of $\mathrm{Mn}$ atoms, the $\mathrm{P}_{2}$ dimers and the layers of sulphur atoms that sandwich the $\mathrm{Mn} / \mathrm{P}_{2}$ layers. There is a van der Waals gap between these sandwich

Send offprint requests to: goossens@rsc.anu.edu.au.

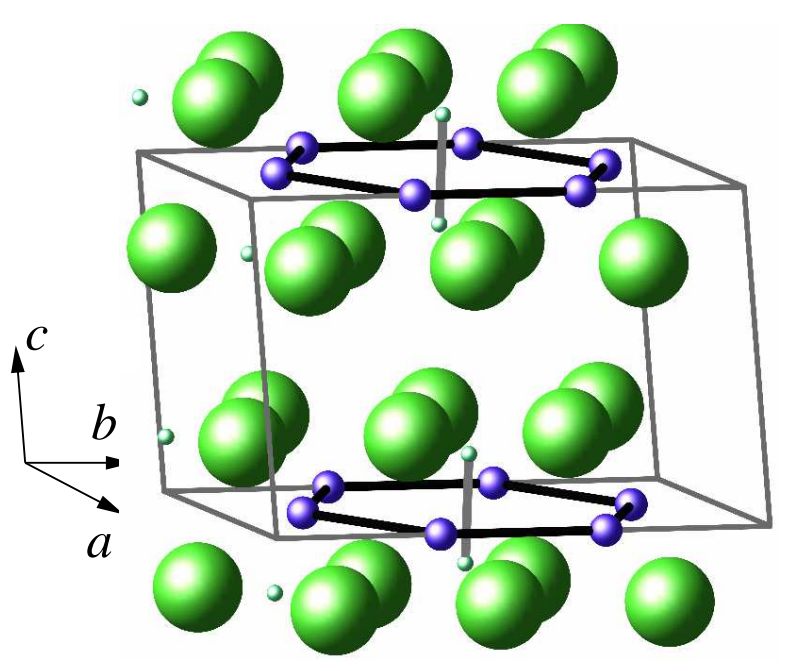

Fig. 1. The crystal structure of $\mathrm{MnPS}_{3}$. Large green spheres are $\mathrm{S}$, blue are $\mathrm{Mn}$ and the $\mathrm{P}_{2}$ pairs are indicted by the dumbbells [11].

structures resulting in very little orbital overlap between atoms on either side of the gap, meaning that both direct exchange and super-exchange are weak, contributing to the 2D magnetic properties and giving the crystals highly anisotropic structural behaviour.

Its experimentally determined magnetic phase diagram has been outlined [12] and its magnetic structure has been explored as a function of applied magnetic field and com- 
position $[13,14]$. They key orderings observed in $\mathrm{MnPS}_{3}$ are that at low $T$ the spins are collinear, perpendicular to the $a b$ planes (that is, they point in the $c^{*}$ direction), antiferromagnetically correlated within the plane (with each spin surrounded by three nearest neighbour spins of opposite sign) and ferromagnetic along $c$. Above $T_{N}(78 \mathrm{~K})$ the system is paramagnetic. When a magnetic field is applied along the spin direction $c^{*}$, a spin flop phase transition takes place [15] such that the spins lie in the $a b$ planes. The magnitude of field required varies with temperature, and when flopped the spins lie (anti)parallel with the $b$ axis [13]. The antiferromagnetic correlations remain unchanged in the spin flop state; it appears to be a rotation of the spin structure from $c^{*}$ to $b$. At $5 \mathrm{~K}$, the spin flop field is found to be $4.7 \mathrm{~T}$ [12]. It might be expected that the ground state of the honeycomb lattice with the dipolar interaction would be non-collinear [16]; however, the system possesses exchange interactions which are much stronger than the dipolar interaction. In this work it is therefore taken that the (isotropic) exchange interactions determine the direction of each spin relative to its neighbours (in other words, hold the system collinear) and the anisotropy then determines the direction of this collinear structure relative to the unit cell axes. This then allows dipolar calculation to be used as a means of determining the preferred spin direction, without the need to calculate the contribution of the exchange, which is taken to be isotropic and therefore will not vary as the spin structure is rotated as a whole. This evolution of the structure is also supported by neutron diffraction measurements which can be well fitted by a collinear spin structure both in the zero field and spin flop states [13].

Very detailed measurements of the spin waves and critical properties of $\mathrm{MnPS}_{3}$ have thrown much light on its behaviour but have not been able to show the nature of the anisotropy governing the phase-transition behaviour $[17,18,6]$. The exchange interactions have also been established, with $a b$-plane exchange values being $J_{1}=-8.9 \mathrm{~K}$ (nearest neighbour (NN); number of such neighbours, $Z_{1}=$ 3 ), $J_{2}=-0.8 \mathrm{~K}$ (second NN, $Z_{2}=6$ ) and $J_{3}=-2.1 \mathrm{~K}$ $\left(3 \mathrm{NN}, Z_{3}=3\right)$. First neighbour exchange between planes is given as $J_{\perp}=0.022 \mathrm{~K}\left(Z_{\perp}=2\right)[17,18,6]$, or $J_{\perp}=$ $0.022 k_{\mathrm{B}} \sim 3 \times 10^{-25} \mathrm{~J}$.

Measurements suggest that below $T_{N}$ the system shows $2 \mathrm{D}$ anisotropic Heisenberg $(2 D A H)$ critical behaviour (with XY-like rather than Ising-like critical exponents), with a crossover to $3 \mathrm{D}$ for $T \gtrsim 0.97 T_{N}$ [18]. The magnetic structure in zero applied field at $5 \mathrm{~K}$ is shown in figure 2 , which shows the antiferromagnetic in-plane correlations. .

As $\mathrm{Mn}^{2+}$ is a spherical ion and expected to show little single-ion anisotropy, dipole-dipole interactions have been considered as mechanisms for the ordering $[19,15]$. It has been shown that this gives rise to ordering perpendicular to the $a b$ plane [15], but also concluded that it cannot be the sole effect, as the observed spin wave gap implies a much smaller anisotropy [6]. Hence it has been shown that there is a single ion anisotropy, $K_{\mathrm{S}}$, weaker than the dipolar and tending to push the spins to lie in the planes. This is most likely a crystal field effect, and in $\mathrm{MnPS}_{3}$ is weaker than the dipolar anisotropy. Here, the dipolar anisotropy is explored in some detail to gain insight into the ordering mechanisms in $\mathrm{MnPS}_{3}$.

\section{The Modelling}

Because the spin structure remains antiferromagnetically correlated, real-space dipolar summation can be performed with good convergence, and a sum over a sphere of radius $250 \AA$ was used (although convergence was tested empirically by performing a handful of extremely large simulations out to over $1000 \AA$ radius). The expression for the potential energy of a pair of magnetic dipoles, $\mu_{1}$ and $\mu_{2}$, in which the distance between north and south poles is negligible compared to the separation, $\mathbf{r}$, is [20]

$$
U=\frac{\mu_{1} \cdot \mu_{2}}{\mathbf{r}^{3}}-\frac{3\left(\mu_{1} \cdot \mathbf{r}\right)\left(\mu_{2} \cdot \mathbf{r}\right)}{\mathbf{r}^{5}}
$$

For SI units this expression is multiplied by $\mu_{0} / 4 \pi$. The dipolar energy of a spin is obtained by summing over all such interactions out to the sum radius and dividing by two, since equation 1 gives the energy of a dipole pair, whereas the final sum is to give the energy of a single dipole in the field caused by all its neighbours.

At low temperatures, $\mu=4.5 \mu_{B}$ [13]. This summation was performed for $3 \mathrm{D}$ systems of spins for a range of values of the parameter $\zeta$, whose definition is outlined below. This allows exploration of the effect of the deviation away from 'ideal' hexagons of the honeycomb lattice.

There are two Mn-Mn 'type' of nearest neighbour vector present in the crystal structure. Figure 2 shows these, labeled $\mathrm{A}$ and $\mathrm{B}$. If they have lengths $d_{\mathrm{A}}$ and $d_{\mathrm{B}}$ then these can be written

$$
d_{\mathrm{A}}=\left(1-2 y_{\mathrm{Mn}}\right)|b|
$$

and

$$
d_{\mathrm{B}}=\sqrt{\left(\frac{|a|}{2}\right)^{2}+\left(\left(\frac{1}{2}-2 y_{\mathrm{Mn}}\right)|b|\right)^{2}}
$$

and the deviation of the honeycomb away from 'ideal' $\left(d_{\mathrm{A}}=d_{\mathrm{B}}\right.$ could be parameterised by the difference or ratio of these two distances, suggesting that a useful definition might be

$$
\zeta=d_{\mathrm{A}} / d_{\mathrm{B}}
$$

$\zeta$ will be driven away from unity by changing $y_{\mathrm{Mn}}$ or by changing $|b| /|a|$.

\subsection{Results of Modelling}

For both the idealised structure $\zeta=1$ and the observed, $\zeta \sim 1.007$, the dipole-dipole energy was found to be a minimum when the spin direction was collinear with $c^{*}$, as is observed in experiment. This minimum was located through a simple, relatively coarse grid search whose parameters were the angles (in spherical coordinates) that 


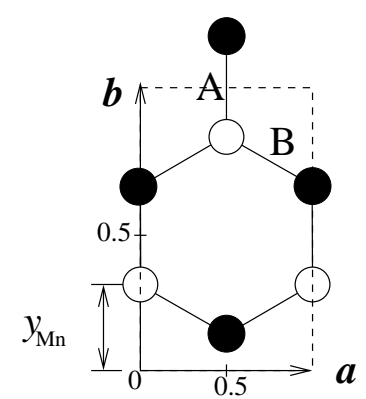

Fig. 2. The two types of inter-ionic vector between Mn atoms in the $a b$ plane, $\mathrm{A}$ and $\mathrm{B}$. In zero applied field and $T<T_{N}$, the magnetic ordering is such that if the filled circles represent spins parallel to $c^{*}$, the open circles represent spins antiparallel to $c^{*}$. The dashed lines and $a$ and $b$ axes outline the $a b$ plane of the unit cell.

the spins made to the $a$ and $c^{*}$ axes. When the approximate position of the minimum had been established by the coarse search, a finer gridded search was performed to locate the minimum more precisely.

The anisotropy energy leads to an estimate of the spin flop field through [21]

$$
B_{\mathrm{sf}}=\sqrt{\frac{2 K}{\chi_{\perp}-\chi_{\|}}}
$$

where $K$ is the anisotropy energy and the susceptibilities are taken from experiment, for example $[12,15]$. Given that the minimum energy configuration occurs when the moments are directed (anti)parallel to $c^{*}$, the dipolar anisotropy preferring the moments to point along $c^{*}$ is here labeled $K_{c^{*}} . K_{c^{*}}$ is taken to be the difference between the internal dipolar energy of the spin configuration calculated with spins parallel to $c^{*}$ and the internal dipolar energy of the spin configuration calculated with spins perpendicular to $c^{*}$ (in other words, in the $a b$ plane) and pointing along the in-plane direction that minimises their energy. In other words, $K_{c^{*}}$ is equal to the difference between the global minimum (pointing along $c^{*}$ ) and the minimum when restricted to the $a b$ plane. $3 \mathrm{D}$ summation leads through equation 5 to $B_{\text {sf }} \sim 8.2 \mathrm{~T}$.

This value is approximately $1.8 B_{\text {sf }}$ (observed) which accords with the idea that there is a second anisotropy pushing the moments into the planes, resulting in a spin flop field less than that calculated. On the assumption that this is a single ion anisotropy of some kind, it is referred to as $K_{\mathrm{S}}$

A second factor is the dependence of the anisotropy energy on $\zeta$. Figure 3 shows a plot of $K_{c^{*}}$ as a function of $\zeta$. It shows that the degree of idealisation of the structure in the calculation has a noticeable if small effect on the magnitude of dipolar anisotropy, but more significantly on the direction the spins would prefer if lying in in the $a b$ plane, as for example in a spinflop transition.

When a sufficiently large magnetic field is applied along $c^{*}$ the spins 'flop' and lie in the $a b$ plane. When this occurs, the spin correlation structure (the arrangement of white

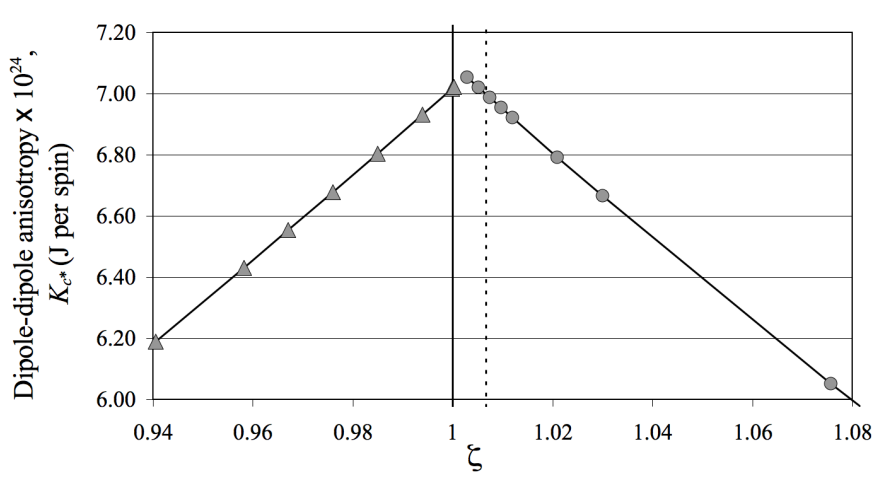

Fig. 3. The dependence of the main dipolar anisotropy, $K_{c^{*}}$, on $\zeta$. For some values the minimum in-plane energy is achieved when spins are along $b$ (circles), for others when spins are along $a$ (triangles). Solid vertical line indicates $\zeta=1$, dashed vertical line indicates $\zeta$ as it is observed in the real structure.

and black circles in figure 2) remains unchanged [13], but now the moments are (anti)parallel to some in-plane direction.

Figure 3 shows that for $\zeta>1$ the lower-energy inplane spin direction is $b$ while for $\zeta<1$, the lower-energy in-plane spin direction is $a$. The dashed line indicates the value of $\zeta$ from experiment [10] and it shows that the moments are expected to prefer the $b$ axis. This is what is experimentally observed [13]. This is strong evidence that the dipole-dipole interaction is indeed governing the nature of the magnetic ordering, at least at temperatures far below $T_{N}$. This also leads to defining a second dipolar anisotropy - the difference in energy between the preferred in-plane ( $a b$ plane) spin direction and the in-plane direction perpendicular to that. This is referred to here as $K_{a b}$. Figure 4 plots $K_{a b}$ against $\zeta$ for a 3D summation.

It has been shown that above $T_{N}$ the system does likely show XY-like critical behaviour, with a crossover to $3 \mathrm{D}$ behaviour very close to $T_{N}$, and that the correlation length follows the Kosterlitz-Thouless behaviour [22]. As the inplane correlation length diverges, the weak inter-plane exchange couplings compound and the system orders in 3D. At the same time, the lengthening in-plane correlations allow the dipolar interaction to come about, as it depends upon the existence of the magnetic long-range order. As soon as the dipolar interaction becomes significant, $K_{c^{*}}$ will align the moments with $c^{*}$, meaning that $K_{a b}$ will be insignificant and will not affect the XY-like nature of the fluctuations. $K_{a b}$ is contingent upon there being moment in the $a b$ plane, which is why it would be very interesting to measure the critical exponents of $\mathrm{MnPS}_{3}$ while applying a strong $(\sim 7 \mathrm{~T})$ magnetic field along $c^{*}$, a field well above the maximum value of the temperature-dependent spin flop field.

It may be considered that the value of $K_{a b}$ indicated by the dashed line in figure 4 is effectively zero, being of magnitude approximately $1 \times 10^{-25} \mathrm{~J} \mathrm{spin}^{-1}$. However, this is of a similar scale to the inter-plane exchange interaction, $J_{\perp}$ as determined by magnon dispersion measurements $[17]$ of $J_{\perp} \sim 3 \times 10^{-25} \mathrm{~J} \mathrm{spin}^{-1}$. Hence at low temperatures 


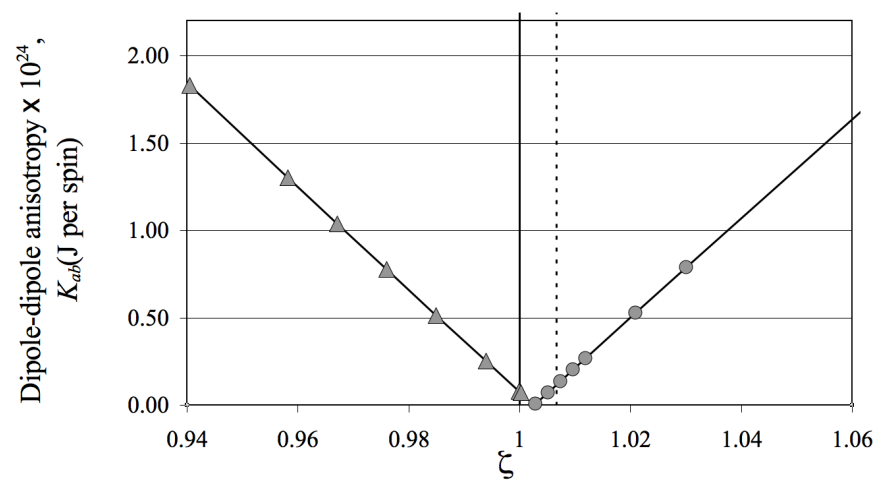

Fig. 4. The anisotropy within the $a b$ plane $\left(K_{a b}\right)$ as it depends on $\zeta$ for a dipole summation performed in 3D.

and when the spinflop forces a large component of the spins into the $a b$ plane, it is reasonable to expect this anisotropy to have some influence - such as selecting the in-plane spin direction, which does indeed seem to be the case.

Single-ion anisotropy terms are typically parameterised by expressions of the form $K_{\mathrm{S}}=D S_{x}^{2}$, where the key aspect here is that $S_{x}$ enters in with power 2 . If the single ion anisotropy in $\mathrm{MnPS}_{3}$ is modelled by a term similar to this which tries to constrain the spins to the $a b$ plane, then in qualitative terms, both it and the dipolar energy will scale, approximately, with the square of the Brillouin function for $S=\frac{5}{2}$ (because $S$ enters into $K_{\mathrm{S}}$ as a square and $\mu$ enters into $K_{c^{*}}$ as $\left.\mu_{1} \cdot \mu_{2}\right)$, then taking $\chi_{\perp}-\chi_{\|}$from experiment $[12,15]$, a plot of the trend of $B_{\mathrm{sf}}$ against $T$ can be constructed (figure 5). This plot shows that the temperature dependence of the two anisotropies appears to give a good model for the $T$ dependence of $B_{\text {sf }}$ for temperatures below $\sim 0.8 T_{N}$. If the summing of $K_{\mathrm{S}}$ and $K_{c^{*}}$ is allowed to explain the magnitude of $B_{\mathrm{sf}}$, then much of the significant behaviour is captured.

As the system warms up experiment shows that the magnitude of $B_{\text {sf }}$ increases [12]. This is largely the result of a decrease in the difference $\chi_{\perp}-\chi_{\|}$. The solid curve in figure 5 suggests that $B_{\mathrm{sf}}$ should start to fall as $T_{N}$ is approached. For pure $\mathrm{MnPS}_{3}$ the critical region proved too narrow for the resolution of the magnetometry to observe this, and the row of solid dots continues to increase. However, the reduction in $B_{\mathrm{sf}}$ as $T$ approached $T_{N}$ has been observed in $\mathrm{Mn}_{x} \mathrm{Zn}_{1-x} \mathrm{PS}_{3}$ for $x=0.8$ and $x=0.5$ [12], suggesting that perhaps it does occur in $\mathrm{MnPS}_{3}$ but over a narrower temperature range than this model suggests.

An attempt has been made to explore the dependence of $K_{c^{*}}$ on pressure. Recent work by Toyoshima et al. [23] has shown that the spin flop field decreases at a rate of $4.0 \times 10^{3} \mathrm{Oe} / \mathrm{GPa}$, or about $8 \% / \mathrm{GPa}$ as pressure is applied. Due to the van der Waals gap, the $c$ axis is expected to change much faster than $a$ or $b$. The microscopic distortion of the unit cell associated with the application of pressure is not known. Therefore three simple models of the effect of pressure on the magnetic anisotropy were initially explored: (1) reduce the length of $c$, leaving other

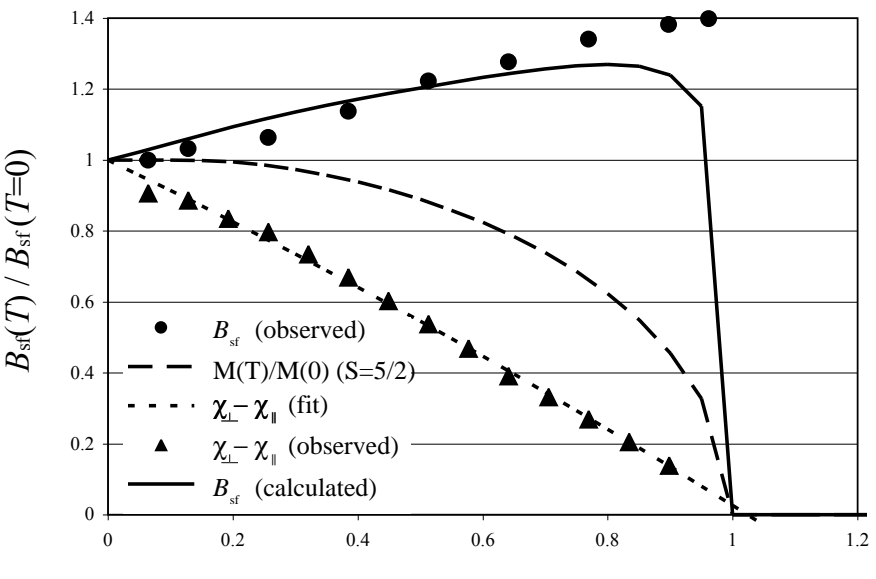

$T / T_{N}$

Fig. 5. The temperature dependence of the spin flop field modelled by using the Brillouin function for $S=\frac{5}{2}$ to give the temperature dependence of the dipolar energy and using the observed temperature dependence of $\chi_{\perp}-\chi_{\|}$.

parameters unchanged (2) reduce $|c|$ and modify $\beta$ such that the $a$-axis component of $c$ is maintained and (3) increase $\beta$.

Of these approaches, (1) and (2) caused the dipolar anisotropy to increase, while (3) caused it to decrease, but very slowly, such that no reasonable increase in $\beta$ could model the magnitude of decrease in $B_{\mathrm{sf}}$ observed in [23].

As a last model, it was posited that pressure might cause $a b$ layers to slide over each other parallel to the $a b$ planes and along the $a$ axis. Such a model was tested by adding a randomly generated offset to the $a$-coordinate of each layer in the structure. Qualitatively, it was found that this could also reduce the dipolar anisotropy, but again the magnitude of reduction was too small.

The inability of any of these structural modifications to give a decrease in the dipolar anisotropy sufficient to explain the results presented in [23] suggests that the pressure is instead influencing the single ion anisotropy. This is quite plausible because if $K_{\mathrm{S}}$ is coming from crystal field effects and the $\mathrm{Mn}^{2+}$ environment is being compressed, which will be relatively easy along $c$, then inter-atomic distances will be decreasing, an effect which is expected to increase the electric field at the $\mathrm{Mn}$ site and increase any single-ion anisotropy. Since $K_{\mathrm{S}}$ favours in-plane spins, this would have the effect of reducing $B_{\mathrm{sf}}$.

\section{Conclusions}

The magnetic ordering observed in $\mathrm{MnPS}_{3}$ at temperatures below $\sim 0.8 T_{N}$ is well explained by the dipole-dipole interaction, which provides a mechanism for preferring the observed low temperature zero field and spin flop spin configurations. Dipolar anisotropy gives a good order of magnitude estimate for the spin flop field, although requires the existence of a second anisotropy because it does overestimate the magnitude of the spin flop field. 
At low temperatures and for small moment deviations away from the $c^{*}$ axis, the system lies in a potential well due to the dipolar anisotropy, and the well is close to isotropic for moment deviations in the $a b$ plane because it is only as the moment components in the plane become large that the in-plane dipolar anisotropy manifests itself. This could easily in concert with the posited XYlike single-ion anisotropy give rise to XY-like (or perhaps anisotropic Heisenberg) critical fluctuations, leaving the $c^{*}$ components of the moments largely unaffected.

Given the deviation of the honeycomb lattice of $\mathrm{Mn}^{2+}$ ions from regularity $(\zeta=1$, see equation 4$)$ and the symmetry in three dimensions of the crystal structure, the dipolar interaction in real $\mathrm{MnPS}_{3}$ can never give a system which is isotropic in the $a b$ plane. There will always be a preferred spin direction, but it only manifests itself when significant moment lies in the $a b$ plane. However, because the moment components in this plane are small unless the spin flop phase is induced, this in-plane anisotropy has little effect on the critical properties. While at temperatures close to $T_{N}$ there will be substantial components in the plane due to thermal fluctuations, the magnetic ordering will not be maintained and so the dipolar energy will not follow the behaviour calculated here, which assumes the observed collinear structure is homogeneously rotated into the plane, something which is known to be true of the spin flop state from neutron magnetic scattering studies.

Measurement of critical scattering in the spin flop phase could shed light on the mechanisms governing order in $\mathrm{MnPS}_{3}$, as in the spin flop state the in-plane anisotropy of the dipolar interaction is manifested, potentially changing the symmetry of the system, which should be observable in the critical behaviour.

\section{Acknowledgments}

The author thanks Dr T. J. Hicks for initiating the research, Dr A. R. Wildes for interesting discussions and the Australian Institute for Nuclear Science and Engineering for funding and the NCI National Facility for computing resources.

\section{References}

1. L. Onsager, Phys. Rev. 65, 117 (1942)

2. R.J. Birgeneau, H.J. Guggenheim, G. Shirane, Phys. Rev. B 1, 2211 (1970)

3. R.A. Cowley, A. Aharony, R.J. Birgeneau, R.A. Pelcovits, G. Shirane, T.R. Thurston, Z. Phys. B 93, 5 (1993)

4. E. Manousakis, Rev. Mod. Phys. 63, 1 (1991)

5. N. Ismail, Y. Temerk, A. El-Meligi, M. Badr, M. Madian, J. Solid State Chem. 183, 984 (2010)

6. A.R. Wildes, H.M. Rønnow, B. Roessli, M.J. Harris, K.W. Godfrey, J. Magn. Magn. Mater. 310, 1221 (2007)

7. H. Zhou, L. Zou, X. Chen, C. Yang, M. Inokuchi, J. Qin, J. Inclusion Phenomena \& Macrocyclic Chem. 62, 293 (2008)

8. A.A. El-Meligi, J. Mater. Sci. Technol. 22, 252 (2006)
9. Y. Kai, S. Xu, Z. Xuan, C. Xingguo, F. Enqin, Q. Jingui, M. Inokuchi, M. Kinoshita, J. Solid State Chem. 177, 4300 (2004)

10. G. Ouvrard, R. Brec, J. Rouxel, Mat. Res. Bull. 20, 1181 (1985)

11. T.C. Ozawa, S.J. Kang, J. Appl. Cryst. 37, 679 (2004)

12. D.J. Goossens, T.J. Hicks, J. Phys.: Condens. Matter 10, 7643 (1998)

13. D.J. Goossens, A.R. Wildes, C. Ritter, T.J. Hicks, J. Phys.: Condens. Matter 12, 1845 (2000)

14. D.J. Goossens, A.J. Studer, S.J. Kennedy, T.J. Hicks, J. Phys.: Condens. Matter 12, 4233 (2000)

15. K. Okuda, K. Kurosawa, S. Saito, M. Honda, Z. Yu, M. Date, J. Phys. Soc. Japan 55, 4456 (1986)

16. V.M. Rozenbaum, Phys. Rev. B 53, 6240 (1996)

17. A.R. Wildes, B. Roessli, B. Lebech, K.W. Godfrey, J. Phys.: Condens. Matter 10, 6417 (1998)

18. A.R. Wildes, H.M. Rønnow, B. Roessli, M.J. Harris, K.W. Godfrey, Phys. Rev. B 74, 094421 (2006)

19. C. Pich, F. Schwabl, J. Magn. Magn. Mater. 148, 30 (1995)

20. W.T. Scott, The Physics of Electricity and Magnetism (John Wiley \& Sons, 1962)

21. A. Tressaud, J.M. Dance, Structure and Bonding 52, 87 (1982)

22. H.M. Rønnow, A.R. Wildes, S.T. Bramwell, Physica B: Condensed Matter 276-278, 676 (2000)

23. W. Toyoshima, T. Masubuchi, T. Watanabe, K. Takase, K. Matsubayashi, Y. Uwatoko, Y. Takano, J. Physics: Conf. Series 150, 042215 (2009) 